\title{
Dronedarone in the management of atrial fibrillation
}

\author{
This article was published in the following Dove Press journal: \\ Open Access Emergency Medicine \\ 27 March 2010 \\ Number of times this article has been viewed
}

\author{
TS Mohamed Saleem' \\ K Bharani \\ C Madhusudhana Chetty \\ K Gauthaman² \\ 'Department of Pharmacology, \\ Annamacharya College of Pharmacy, \\ New Boyanapalli, Rajampet, \\ Andhrapradesh, India; 'Department of \\ Pharmacognosy, Himalayan Pharmacy \\ Institute, Majhitar, Sikkim, India
}

\begin{abstract}
Atrial fibrillation is the most common type of tachyarrhythmia caused by multiple re-entrant wave forms within the atria and bombarding the atrioventricular node several times making it beat in a rapid, disorganized fashion termed "fibrillation". In atrial fibrillation, atria beat more than 300 times per minute. The arrhythmatous condition needs to be controlled, as humans cannot withstand this rapid and chaotic beating of the heart. New investigational drugs like Dronedarone ${ }^{\circledR}$ are being used. Dronedarone is the most recent antiarrhythmic drugs. It was approved by US-FDA on July 2nd 2009 and is available in the USA as Multaq tablets (400 mg). Dronedarone falls under the category of multiple ion channel blocker. It mainly targets the repolarization currents, making them less active and hence prolonging the action potential duration (APD). Dronedarone also exhibits antiadrenergic activity, thus reducing the pace of the pacemaker. Dronedarone has been proven to be a safer and efficacious AAD, evidenced by both animal and human studies. These studies showed that there was prolongation of the APD and absence of QT interval prolongation with long term administration of the drug. Also there was reduced thyroid hormone receptor expression. Dronedarone is significantly safer and effective in maintaining the sinus rhythm and reducing the ventricular proarrhythmias, justifying it for the long term treatment of atrial fibrillation compared to other antiarrhythmic drugs.
\end{abstract}

Keywords: dronedarone, atrial fibrillation, ion channel blocker, antiarrhythmic drugs, multaq

\section{Introduction}

Atrial fibrillation (AF) is the most common of the serious cardiac rhythm disturbances and is responsible for substantial morbidity and mortality. The prevalence of AF is almost $1 \%$ and it is estimated that by the year 2050, approximately 5 million subjects will have AF in the USA. There are currently two major treatment strategies for AF: rate control and rhythm control. Sustained sinus rhythm is associated with an improved quality of life and improved exercise performance. ${ }^{1} \mathrm{AF}$ is the most common type of tachycardia in humans. ${ }^{2-4} \mathrm{AF}$ prevalence increases with age..$^{5}$ It typically occurs in the setting of specific structural and electrophysiological changes in the atria, which contribute to the perpetuation of arrhythmia by atrial remodeling. ${ }^{6}$

The rapid atrial activation, as occurs with AF, leads to the progressive shortening of the atrial action potential duration (APD), due to the down regulation of the L-type calcium currents and increase in the inward rectifier potassium currents. ${ }^{7-9}$ The shortened APD allows for smaller re-entry circuits and acceleration or stabilization of the re-entrant rotors helping to perpetuate arrhythmia. ${ }^{10,11}$ According to the leading circle hypothesis, maintenance of the re-entry depends on the wavelength or the distance travelled by an impulse during its own effective refractory period (ERP). ${ }^{12}$
Correspondence: TS Mohamed Saleen Annamacharya College of Pharmacy, New Boyanapalli, Rajampet-516I26, Andhrapradesh, India Tel +9l 9701978543

Email saleemcology@gmail.com 
From a clinical point of view, AF is usually classified into three categories: paroxysmal, persistent, and permanent AF. In paroxysmal type, the episodes of AF spontaneously terminate after no more than a couple of days; in the persistent type the sustained arrhythmic episodes do not terminate spontaneously but require electrical or pharmacological cardioversion for a normal sinus rhythm to be restored and finally as the disease evolves AF becomes permanent and cannot be terminated. The fourth type is lone AF; it has been used to describe AF in individuals without structural or cardiac or pulmonary disease, with low risk of thrombo-embolism. One of the main characteristics of $\mathrm{AF}$ is its propensity for recurrence. The basic and clinical studies have both shown that AF can lead to AF; the "AF begets AF" phenomenon. ${ }^{13}$

Currently amiodarone is one of the most widely used and most effective antiarrhythmic drugs (AADs) with little proarrhythmic potential. However, during chronic usage amiodarone and its active metabolite desethylamiodarone can cause serious extra cardiac adverse effects, including effects on the thyroid. Therefore, a safer and effective antiarrythmic agent needs to be developed. ${ }^{1}$ Hence, there is an immense need of new drugs for AF therapy as the traditional treatment has many unwanted effects, especially proarrhythmia. Dronedarone is one such new investigational drug that has been proven to be safer and efficacious compared to other AADs. The present review mainly focuses on the pharmacodynamics and pharmacokinetics and clinical aspects of dronedarone. In this respect the review will improve the basic idea to understand the pharmacology of dronedarone. This is useful for the further research in cardiovascular disease particularly in AF.

\section{Dronedarone}

Dronedarone (Figure 1) is a newer benzofuran derivative with a structural resemblance to amiodarone (Figure 2), with two molecular changes: it lacks the iodine moiety and it has a methane sulfonyl group that decreases lipophilicity, resulting in a shorter half-life and lower tissue accumulation. ${ }^{1,14}$ It falls under the category of a multichannel blocker. The synthesis of dronedarone is based on the amiodarone molecule and chemically it is [N-(2-butyl-3-\{4-(3-(dibutylamino) propoxy)-benzyl benzifuran-5-yl\}methansulfonamide]. It is structurally modified to avoid toxicity risks. ${ }^{15}$

Unlike most other AADs, Dronedarone has been found to be safe, well tolerated and nonproarrhythmic in the AF population. ${ }^{16-18}$ This is mainly due to the removal of iodine molecules with the goal of eliminating the thyroid and pulmonary toxicities. In addition, the addition of a methyl

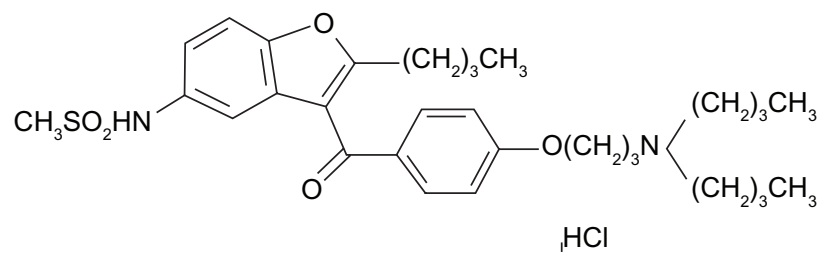

Figure I Structure of dronedarone.

sulphonyl group to decrease the lipophilicity there by reduces tissue accumulation. ${ }^{17}$

\section{Pharmacokinetic parameter}

Due to the extensive presystemic or first pass metabolism, the absolute bioavailability of dronedarone was found to be very low, where it is only about $\sim 15 \%$, because of its high first pass metabolism. ${ }^{19}$ The peak plasma concentration after the oral administration of the drug is reached within 3-6 hours. Dronedarone is extensively metabolized by CYP3A4 isoenzymes. Initially it includes N-debutylation to form the active $\mathrm{N}$-debutyl metabolite that exerts the pharmacodynamic effects. ${ }^{19}$ The steady-state serum level of dronedarone is achieved in five to seven days. The elimination half-life of dronedarone is 24 hours. It is cleared by a nonrenal mechanism. Like amiodarone, oral bioavailability is increased two- to threefold when taken with food. It is well absorbed after oral administration ( $70 \%$ to $100 \%$ ). The bioavailability is relatively low $(15 \%)$ due to extensive hepatic first-pass metabolism by cytochrome P450 (CYP) 3A4 and CYP2D6, thus requiring twice-daily dosing to achieve steady-state serum levels. ${ }^{20}$

\section{Pharmacodynamic parameter}

The atrial action potential is the result of a coordinated activation of several voltage-activated depolarization (inward) and repolarizing (outward) currents. The resting membrane potential of atrial myocytes is controlled by the inward rectifying potassium currents $\left(\mathrm{I}_{\mathrm{Ki}}\right)$ and by the muscarinic potassium currents $\left(\mathrm{I}_{\mathrm{KAch}}\right)$. The inward rectifying potassium current appears to be less prominent in the atria than in the ventricles, the muscarinic potassium current is only expressed in the atria. This difference may explain the enhanced sensitivity of the atrial myocytes to the neurohormonal modulation and this also acts as the basis of the drug treatment specific for the atria. ${ }^{21}$ Different ion channels are found in the atrial tissue compared to the ventricular tissue. AADs that have specific activity for ion channels found exclusively or predominantly in the atria may provide effective treatment for $\mathrm{AF}$ without ventricular 


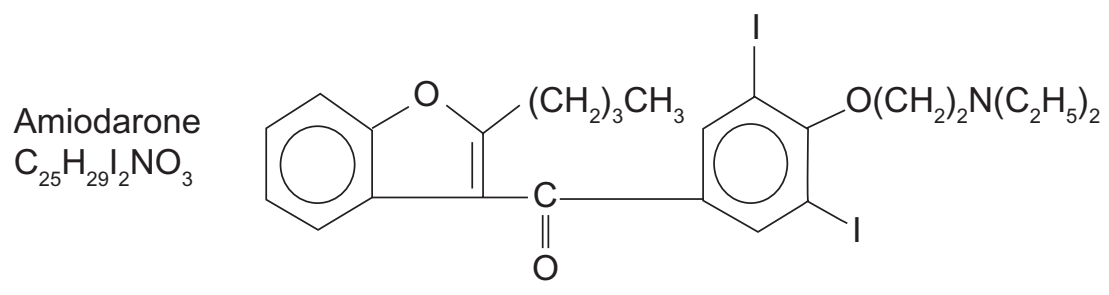

Figure 2 Structure of amiodarone.

proarrhythmia. Several such ion channels are attractive targets for the treatment of AF. In the case of AF, there is a manifestation of the shortening of APD, with a relatively greater contribution from the ultra rapid potassium current $\mathrm{I}_{\mathrm{Kur}}$, the transient outward potassium current $\mathrm{I}_{\text {to }}$, and the muscarinic acetylcholine potassium current. As well, there is a decreased influence of the delayed rectifying potassium currents (of both rapid and slow type) and calcium currents (Figures 3, 4). ${ }^{22}$

The currently available AADs mainly target the potassium channels and tend to block the late phase 3 repolarization currents, $\mathrm{I}_{\mathrm{Kr}}$ and $\mathrm{I}_{\mathrm{Ks}}$ and may make them less active during $\mathrm{AF}^{22}$ Dronedarone functions as a blocker of multiple ion channels and exhibits noncompetitive antiadrenergic activity. ${ }^{23}$ Dronedarone reversibly inhibits a set of potassium currents, $\mathrm{I}_{\mathrm{CaL}}$, sodium-calcium exchanger and sodium currents. Heterogenity is observed among various cardiac regions (left vs right ventricular and Purkinje fibres) in response to the drug. Dronedarone leads to no change or even shortening of the APD in most regions of the left ventricular and purkinje cells. ${ }^{24}$

\section{Acute ion channel-blocking properties dronedarone in-vitro}

Many ion channel-blocking properties of dronedarone have been studied under in vitro conditions using the cells of various species. Different half maximal inhibitory concentration $\left(\mathrm{IC}_{50}\right)$ values have been observed for various species and these values differ accordingly when the target and the type of cell species has been changed. The $\mathrm{IC}_{50}$ values of dronedarone for muscarinic potassium currents of rabbit sinus node cells and Guinea pig atrial cells were found to be $63 \mathrm{nmol} / \mathrm{L}$ and $10 \mathrm{nmol} / \mathrm{L}$ respectively. ${ }^{25,26} \mathrm{The}^{\mathrm{IC}_{50}}$ value of dronedarone was found to be $\sim 9 \mu \mathrm{mol} / \mathrm{L}$ when the target was $\mathrm{I}_{\text {HERG }}$ in Xenopus oocytes at room temperature. ${ }^{27}$ The $\mathrm{IC}_{50}$ value was found to be high, $\sim 180 \mathrm{nmol} / \mathrm{L}$, when the target was L-type calcium currents in guinea pig ventricular myocytes recorded at $35 \pm 1{ }^{\circ} \mathrm{C} .{ }^{28}$ There is about $97 \%$ reduction in the production of sodium currents with $3 \mu \mathrm{mol} / \mathrm{L}$ using the human atrial myocytes recorded at room temperature. ${ }^{29}$

\section{Adverse events}

Like any other drugs, dronedarone also produces adverse events but are low risk compared to other AADs. These include: diarrhea, nausea, bradycardia, QT prolongation syndrome, and rash. It is also necessary to let your health care provider know if you develop any heart failure symptoms or worsening heart failure while on dronedarone. Such symptoms may include shortness of breath, wheezing, difficulty in sleeping due to breathing problems, rapid weight gain, and water retention or swelling of the arms, legs, feet, or hands. ${ }^{30}$

\section{Contraindications}

Dronedarone is contraindicated ${ }^{31}$ in patients with:

- NYHA class IV heart failure or NYHA class II/III heart failure with a recent decompensation requiring hospitalization.

- Second or third degree atrioventricular block or sick sinus syndrome.

- Bradycardia of less than 50 beats/min.

- Concomitant use of strong CYP3A inhibitors, such as ketoconazole, itraconazole, and voriconazole.

- Concomitant use of drugs or herbal products that prolong the QT interval, and might increase the risk of torsadede-pointes.

- Severe hepatic impairment in pregnancy, as dronedarone is a category $\mathrm{X}$ drug and hence it may produce the harmful effects in the fetus.

\section{Drug interactions}

Dronedarone is primarily metabolized by CYP3A and is a moderate inhibitor of CYP3A and CYP2D6. ${ }^{31}$ The blood levels of dronedarone can therefore be affected by inhibitors and inducers of CYP3A and can also interact with drugs that are the substrates of CYP3A and CYP2D6. Dronedarone has been found to interact with drugs similarly to some phenothiazones, 


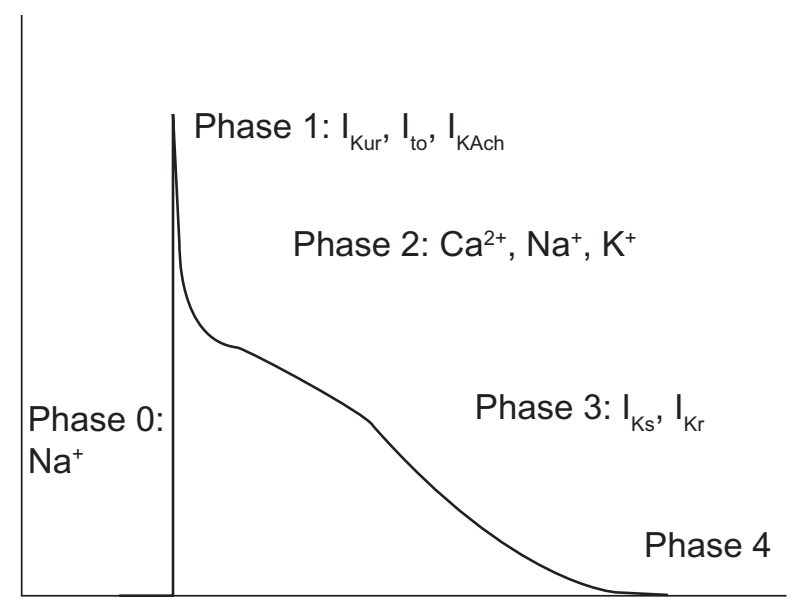

Figure 3 The five phases of the cardic myocyte action potential.

certain macrolides, some class I and class III AADs that prolong the QT interval and hence induce torsades-de-pointes.

1. Where it potentiates the electrophysiologic effects of dronedarone, such as decreased atrioventricular (AV) node conduction. dronedarone was found to increase the levels of digoxin when co-administered.

2. Calcium channel blockers with depressant effects on sinus and $\mathrm{AV}$ nodes could potentiate the dronedarone effects on conduction.

3. When $\beta$-blockers were administered with dronedarone, severe bradycardia was observed. Hence a low dose of $\beta$-blockers is given initially.

4. Repeated administration of CYP3A inhibitors such as ketoconazole resulted in a 17 -fold increase in the dronedarone exposure and hence they are contraindicated.

5. Grape fruit juice, a moderate inhibitor of CYP3A, resulted in a threefold increase in dronedarone exposure. Patients should avoid grape fruit beverages while on dronedarone.

6. Dronedarone was also found to interact with many statins such simvastatin and increases exposure and so statins are also contraindicated.

7. In patients with $\mathrm{AF} /$ atrial flutter, there was no observed excess risk of bleeding when dronedarone was co-administered with the oral anticoagulants and CYP2C9 substrates, warfarin and losartan. No interactions were observed between dronedarone and losartan.

8. Theophylline, a CYP1A2 substrate, did not show an increase in the steady state exposure when co-administered with dronedarone.

Although some possible interactions are mentioned, along with their contraindications, the actual mechanism of interactions has not yet being elucidated for dronedarone.

\section{Evidence from animal studies}

Dronedarone has similar acute and long-term electrophysiologic properties to amiodarone in rabbit atrial muscle. ${ }^{32}$ It was also found that dronedarone produced less prolongation of ventricular repolarization than amiodarone during long-term treatment of canines. The lack of APD prolongation with short term administration in animal models may relate to the balanced influence on depolarizing and repolarizing currents. ${ }^{24}$ In the rabbit atrial muscle, long-term (four weeks) oral dronedarone administration of $100 \mathrm{mg} / \mathrm{kg} /$ day led to the prolongation of the APD to $\sim 90 \%$ repolarization by $\sim 19 \%$, while short term administration shortened the $\mathrm{APD}_{90}$ which was found to be similar to amiodarone in the same model. ${ }^{32} \mathrm{In}$ a similar model of the rabbit atrial muscle, dronedarone was evaluated in a five-week oral administration for the effects on ventricular $\mathrm{APD}_{90}$. In this study, dronedarone $100 \mathrm{mg} / \mathrm{kg} /$ day produced a greater prolongation of APD at a cycle length of $300 \mathrm{~ms}$. This prolongation of the ventricular APD also translated into a prolongation of the QT syndrome of conscious rabbits $\sim 30 \%$ increase, ie, from $140 \pm 9$ to $183 \pm 9 \mathrm{~ms}$ for dronedarone $100 \mathrm{mg} / \mathrm{kg} /$ day. ${ }^{33}$

Results obtained in a canine model of chronic atrioventricular block showed a small but significant increase in the QT interval with long term oral dronedarone administration for three weeks ( $20 \mathrm{mg} / \mathrm{kg}$ twice daily). ${ }^{34}$ In contrast, a study in healthy dogs fed with $25 \mathrm{mg} / \mathrm{kg}$ (twice daily) dronedarone for four weeks demonstrated the absence of QT interval prolongation, whereas amiodarone led to the significant QT interval prolongation. ${ }^{24}$ Differences in the thyroid hormone-dependent gene expression between amiodarone and dronedarone have been noted in rat hearts. Thyroid receptor $\left(\mathrm{TR}_{\alpha}\right)$ expression was reduced by amiodarone and dronedarone in the right atrium, whereas expression of $\mathrm{TR}_{\alpha 1}$ was increased with amiodarone but not with dronedarone in the left ventricular apex. ${ }^{35}$ It was also observed in a dog model of acquired long QT syndrome caused long term atrioventricular block, torsades-de-pointes occurred more frequently with dronedarone than amiodarone. ${ }^{36}$

\section{Evidence from human studies}

Several prospective, randomized, double-blind, multicenter clinical trials of dronedarone have been performed. These include the following trials for products, such as Dose Adjustment For Normal Eating (DAFNE), Erasmus University Research Institute for Decision Information System (EURIDIS), American-Australian-African trial with DronedarONe In atrial fibrillation or flutter patients for the maintenance of Sinus rhythm (ADONIS), Antiarrhythmic Trial 


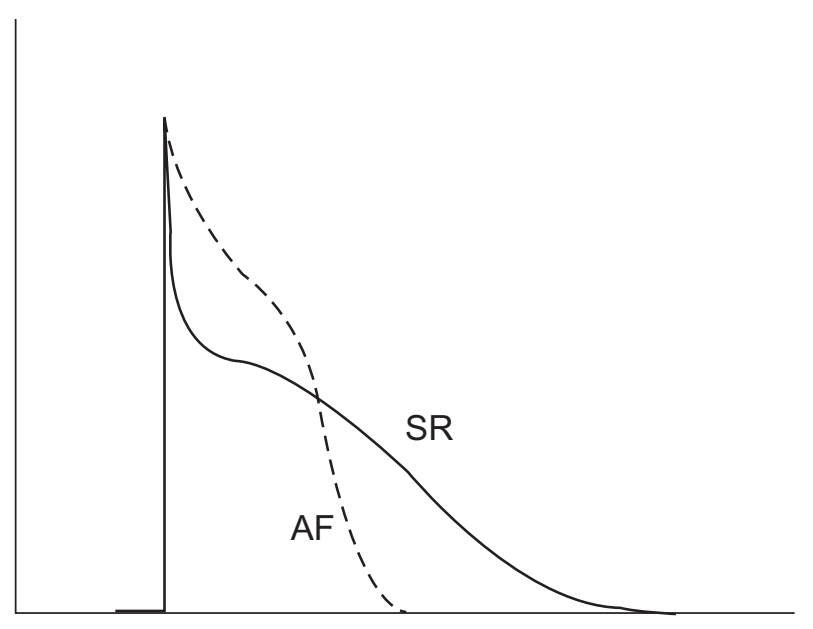

Figure 4 Controlled action potential versus remodeled action potential in AF. Abbreviations: AF, atrial fibrillation; $S R$, sinus rhythm.

with Dronedarone in Moderate-to-Severe Congestive Heart Failure Evaluating Morbidity Decrease (ANDROMEDA), Efficacy and safety of dRonedArone for The cOntrol of ventricular rate during atrial fibrillation (ERATO), A placebocontrolled, double-blind, parallel arm Trial to assess the efficacy of dronedarone $400 \mathrm{mg}$ bid for the prevention of cardiovascular Hospitalization or death from any cause in patiENts with Atrial fibrillation/atrial flutter (ATHENA), Efficacy and Safety of Dronedarone Versus Amiodarone for the Maintenance of Sinus Rhythm in Patients With Atrial Fibrillation (DIONYSOS).

\section{DAFNE}

DAFNE is a dose-finding trial, where the safety and effectiveness of dronedarone at a dosage of $400 \mathrm{mg}$ twice daily was measured. It evaluated dronedarone $800 \mathrm{mg}, 1200 \mathrm{mg}$, and $1600 \mathrm{mg} /$ day for AF relapse population in 270 patients with persistent AF. A six-month follow-up was observed, where the time to AF relapse increased in dronedarone patients, with a median of 60 days compared with five days on placebo. At the end of the six-month follow up, $25 \%$ of the patients receiving dronedarone $800 \mathrm{mg}$ /day remained in a sinus rhythm. It was also found that there is potentially a higher rate of gastrointestinal adverse effects among the patients assigned to $1200 \mathrm{mg} /$ day and $1600 \mathrm{mg}$ /day. This led to discontinuation of the drug. ${ }^{19}$

\section{EURIDIS and ADONIS}

These were two identically designed prospective trials evaluating the efficacy of dronedarone for sinus rhythm maintenance, including a total of 1237 patients with paroxysmal or persistent AF or atrial flutter. Patients were randomized to receive either dronedarone $(400 \mathrm{mg}$ ) or placebo twice daily for one year. The results were observed so, as a median time to the first episode of AF or flutter of 53 days in the placebo group compared with 116 days in the dronedarone group $(P<0.0001){ }^{18}$

\section{ANDROMEDA}

The ANDROMEDA trial was intended as a survival trial evaluating the dronedarone treatment $(400 \mathrm{mg} /$ day twice daily) compared with placebo in patients with placebo in patients with the symptomatic heart failure and systolic left ventricular dysfunction (without AF). This trial was terminated because of increased mortality with dronedarone after the inclusion of 617 patients and median treatment duration of approximately two months. Twenty-five patients $(8 \%)$ in the dronedarone group died, compared to 12 in the placebo group $(4 \%)$. It was suggested that the deaths were predominantly due to worsening heart failure, and also there was no evidence of proarrhythmia in the dronedarone group. Increase in the serum creatinine levels were observed more frequently in the active treatment arm. Since dronedarone causes a reversible increase in the serum creatine levels, this could potentially have been mistaken for angiotensin-converting enzyme (ACE) inhibitor or angiotensin-II receptor antagonist toxicity which may cause worsening of the heart failure. This is one of the possible explanations for the increased mortality observed with dronedarone therapy in ANDROMEDA. As a result of this trial, dronedarone is not recommended for the use in patients with a history of New York Heart Association Classification (NYHA) III/IV heart failure. ${ }^{37}$

\section{ERATO}

ERATO was the only dronedarone trial that enrolled patients with permanent $\mathrm{AF}$ to evaluate the rate control properties with dronedarone in addition to the standard rate control therapy. This trial found that dronedarone significantly reduced the mean 24-hour ventricular rate by 12 beats/minute vs placebo $(P<0.0001){ }^{38}$

\section{ATHENA}

Completed in January 2008, ATHENA is the most recent study of dronedarone. The trial included 4628 patients with paroxysmal or persistent AF and was designed as an outcome trial. It is the largest antiarrhythmic drug trial in AF conducted to date. ATHENA observed a $24 \%$ reduction in the risk of cardiovascular hospitalization or death during a follow-up of nearly 21 months with regard to the primary end point (death from any cause or cardiovascular 
hospitalization). The population studied included elderly AF patients (mean age, $72 \pm 9$ years) with additional risk factors. Interestingly there were several potentially important findings regarding the secondary end point. Cardiovascular mortality was reduced by $29 \%$ in patients receiving dronedarone. Dronedarone-treated patients were hospitalized less often for cardiovascular reasons (25\% less). The death rate from cardiac arrhythmia was greatly reduced by dronedarone treatment ( $45 \%$ risk reduction). ${ }^{39}$

\section{Conclusion}

Atrial fibrillation is a growing medical problem with increasing prevalence. Hence, improved therapeutic approaches are needed, among which dronedarone may prove useful, especially in the long-term treatment of AF with fewer adverse effects. Many other drugs like propafenone SR, azimilide, tedisamil, other atrial selective drugs such as AZD7009, AVE0118, and some serotonin- $5 \mathrm{HT}_{4}$ receptor antagonists (which reduce the calcium load in the myocardial cells) are under investigation for the treatment of AF. Although attempts are made to cure AF by catheter ablation techniques, it remains questionable whether this technique will ever be applicable to the majority of the patients. Dronedarone proves to be safe and effective for all types of AF and for all the age groups. It is likely that various forms of AF therapy will optimally be tailored to specific patient populations. This is an area of major ongoing investigation that provides hope for major improvements in AF management over the next decade.

\section{Disclosures}

The authors declare no conflicts of interest.

\section{References}

1. Pawan DP, Rohit B, Dipal DP, Bhaskar LA, Param PS, Rohit RA. Dronedarone for atrial fibrillation: a new therapeutic agent. Vasc Health Risk Manag. 2009;5:635-642.

2. Ezekowitz MD. Atrial fibrillation: The epidemic of the new millennium. Ann Intern Med. 1999;131(7):537-538.

3. Wellens HJ. Atrial fibrillation-the last big hurdle in treating supraventricular tachycardia. N Engl J Med. 1994;331(14):944-945.

4. Lévy S, Breithardt G, Campbell RW, et al. Atrial fibrillation: Current knowledge and recommendations for the management. Working Group on Arrhythmias of the European Society of Cardiology. Eur Heart J. 1998;19(9):1294-1320.

5. Feinberg WM, Blackshear JL, Laupacis A, Kronmal R, Hart RG. Prevalence, age distribution and gender of patients with atrial fibrillation. Analysis and implications. Arch Intern Med. 1995;155(5):469-473.

6. Haïssaguerre M, Jaïs P, Shah DC, et al. Spontaneous Initiation of Atrial fibrillation by ectopic beats originating in the pulmonary veins. $N$ Engl J Med. 1998;339(10):659-666.

7. Nattel S. New ideas about atrial fibrillation 50 years on. Nature. 2002;415(6868):219-226.

8. Qi XY, Yeh YH, Xiao L, et al. Cellular signalling underlying atrial tachycardia remodelling of the L-type calcium currents. Cir Res. 2008;103(8):845-854.
9. Cha TJ, Ehrlich JR, Chartier D, Qi XY, Xiao L, Nattel S. K -3 based inward rectifier potassium currents: Potential role in atrial tachycardia remodelling effects on atrial repolarization and arrhythmias. Circulation. 2006;113(14):1730-1737.

10. Pandit SV, Berenfeld O, Anumonwo JM, et al. Ionic determinants of functional re entry in a 2-D model of human atrial cells during simulated chronic atrial fibrillation. Biophys J. 2005;88(6):3806-3821.

11. Jalife J, Berenfold O, Mansour M. Mother, rotors and fibrillatory conduction: A mechanism of atrial fibrillation. Cardiovasc Res. 2002;54(2):204-216.

12. Allessie MA, Bonke FI, Schopman FJ. Circus movement in rabbit atrial muscle as a mechanism of tachycardia, III: The leading circle concept: A new model of circus movement in cardiac tissue without the involvement of an anatomic obstacle. Cir Res. 1977;41(1):9-18.

13. Wijffels MC, Kirchhof CJ, Dorland R, Allessis MA. Atrial fibrillation begets atrial fibrillation: A study in awake chronically instrumented Goats. Circulation. 1995;92(7):1954-1968.

14. Van Beeren HC, Jong WM, Kaptein E, Visser TJ, Bakker O, Wiersinga WM. Dronerarone acts as a selective inhibitor of 3,5,3'-triiodothyronine binding to thyroid hormone receptor-alpha1: in vitro and in vivo evidence. Endocrinology. 2003;144(2):552-558.

15. Wegener FT, Ehrlich JR, Hohnloser SH. Dronedarone; an emerging agent with rhythm and rate controlling effects. J Cardiovascular Electrophysiol. 2006;17(Suppl 2):S17-S20.

16. Ehrlich JR, Nattel S. Novel approaches for pharmacological management of atrial fibrillation. Drugs. 2009;69(7):757-774.

17. Zareba KM. Dronedarone: A new anti-arrhythmic agent. Drugs Today (Barc). 2006;42(2):75-86.

18. Singh BN, Connolly SJ, Crijns HJ, et al; EURIDIS and ADONIS Investigators. Dronedarone for maintenance of sinus rhythm in atrial fibrillation or flutter. N Engl J Med. 2007;357(10):987-999.

19. Touboul P, Brugada J, Capucci A, Crijns HJ, Edvardsson N, Hohnloser SH. Dronedarone for the prevention of atrial fibrillation: A dose-ranging study. Eur Heart J. 2003;24(16):1481-1487.

20. Han TS, Williams GR, Vanderpump MP. Benzofuran derivatives and the thyroid. Clin Endocrinol (Oxf). 2009;70(1):2-13.

21. Bril A. Recent advances in arrhythmia therapy: Treatment and prevention of atrial fibrillation. Curr Opin Pharmacol. 2002;2(2):154-159.

22. Morrow JP, Reiffel JA. Drug therapy for atrial fibrillation: what will its role be in the era of increasing use of catheter ablation? Pacing Clin Electrophysiol. 2009;32(1):108-118.

23. Celestino D, Medei E, Moro S, Elizari MV, Sicouri S. Acute in-vitro effects of Dronedarone, an iodine-free derivative, and amiodarone, on the rabbit sinoatrial node automaticity: A comparitive study. J Cardiovas Pharmacol Ther. 2007;12(3):248-257.

24. Varró A, Takács J, Németh M, et al. Electrophysiological effects of Dronedarone (SR 33589), a non-iodinated amiodarone derivative in the canine heart: Comparison with amiodarone. Br J Pharmacol. 2001;133(5):625-634.

25. Altomare C, Barbuti A, Viscomi C, Baruscotti M, DiFrancesco D. Effects of dronedarone on acetylcholine activated current in rabbit SAN cells. Br J Pharmacol. 2000;130(6):1315-1320.

26. Guillemare E, Marion A, Nisato D, Gautier P. Inhibitory effects of dronedarone on muscarinic $\mathrm{K}^{+}$current in Guinea pig atrial cells. J Cardiovas Pharmacol. 2000;36(6):802-805.

27. Thomas D, Kathofer S, Zhang W, et al. Acute effects of Dronedarone on both components of the cardiac delayed rectifier $\mathrm{K}^{+}$current, HERG KvLQT1/minK potassium channels. Br J Pharmacol. 2003;140(5): 996-1002.

28. Gautier P, Guillemare E, Marion A, Bertrand JP, Tourneur Y, Nisato D. Electrophysiologic characterisation of Dronedarone in Guinea pig ventricular cells. J Cardiovas Pharmacol. 2003;41(2):191-202.

29. Lalevée N, Nargeot J, Barrére-Lemaire S, Gautier P, Richard S. Effects of amiodarone and dronedarone on voltage dependent sodium current in human cardiomyocytes. J Cardiovas Electrophysiol. 2003;14(8):885-890.

30. Dronedarone side effects. 2010. Available from: http://www.ganfyd. org/index.php/title-dronedarone. Accessed on February 11, 2010. 
31. RxList. Multaq: Contraindications. July 20, 2009. Available from: http://www.rxlist.com/multaq-drug.htm. Accessed on February 11, 2010 .

32. Sun W, Sarma JS, Singh BN. Chronic and acute effects of dronedarone on the action potential of rabbit atrial muscle preparations: Comparison with amiodarone. J Cardiovas Pharmacol. 2002;39(5):677-684.

33. Sun W, Sarma JS, Singh BN. Electrophysiological effects of dronedarone (SR 33589), a non-iodinated benzofuran derivative in the rabbit heart: comparison with amiodarone. Circulation. 1999;100(22):2276-2281.

34. Verduyn SC, Vos MA, Leunissen HD, van Opstal JM, Wellens HJ. Evaluation of the acute electrophysiologic effects of intravenous Dronedarone, an amiodarone like agent with special emphasis on ventricular repolarization and acquired torsades-de-pointes arrhythmias. J Cardiovas Pharmacol. 1999;33(2):212-222.

35. Stoykov I, van Beeren HC, Moorman AF, Christoffels VM, Wiersinga WM, Bakker O. Effect of amiodarone and dronedarone administration in rats on thyroid hormone dependent gene expression in different cardiac components. Eur J Endocrinol. 2007;156(6):695-702.
36. van Opstal JM, Schoenmakers M, et al. Chronic amiodarone evokes no torsade de pointes arrhythmias despite QT lengthening in an animal model of acquired long-QT syndrome. Circulation. 2001;104(22):2722-2727.

37. Køber L, Torp-Pedersen C, McMurray JJ, et al; Dronedarone Study Group. Increased mortality after Dronedarone therapy for severe heart failure. N Engl J Med. 2008;358(25):2678-2687.

38. Davy JM, Herold M, Hoglund C, et al; ERATO Study Investigators. Dronedarone for the control of ventricular rate in permanent atrial fibrillation: the Efficacy and safety of dronedarone for the control of ventricular rate during atrial fibrillation (ERATO) study. Am Heart J. 2008;156(3):527.e1-e9.

39. Hohnloser SH, Crijns HJ, van Eickels M, et al; ATHENA Investigators. Effect of Dronedarone on cardiovascular events in Atrial fibrillation. N Engl J Med. 2009;360(7):668-678.

\section{Publish your work in this journal}

Open Access Emergency Medicine is an international, peer-reviewed, open access journal publishing original research, reports, editorials, reviews and commentaries on all aspects of emergency medicine. The manuscript management system is completely online and includes a very quick and fair peer-review system, which is all easy to use.

\section{Dovepress}

Visit http://www.dovepress.com/testimonials.php to read real quotes from published authors. 